\title{
Promotion of deflagration-to-detonation transition by repeated obstacle rods
}

\author{
Yoko SEKI*, Ryuji KOBAYASHI*, Wookyung KIM*, Tomoyuki JOHZAKI* and Takuma ENDO* \\ *Mechanical Engineering Program, Hiroshima University \\ 1-4-1 Kagamiyama, Higashi-Hiroshima, Hiroshima 739-8527, Japan \\ E-mail: takumaendo@hiroshima-u.ac.jp
}

Received: 16 September 2020; Revised: 8 December 2020; Accepted: 6 January 2021

\begin{abstract}
We experimentally investigated the promotion of deflagration-to-detonation transition (DDT) in hydrogen-air mixtures contained in a tube in which straight-shaped rods were installed as obstacles. In the experiments, the number of obstacle rods, their spacing, their blockage ratio, and the equivalence ratio of the hydrogen-air mixture were varied as the governing parameters. The obstacle rods had a spacing of 10 or $20 \mathrm{~mm}$ and a blockage ratio of $0.32,0.41$, or 0.51 . As a result of an optimization of the obstacle-rod conditions, when fourteen rods, whose blockage ratio was 0.32 , were installed in a tube with a spacing of $20 \mathrm{~mm}$ and with a hydrogen-air mixture with equivalence ratios from 0.8 to 1.2 , the run-up distance to the DDT was shortened to approximately 20 times the tube diameter.
\end{abstract}

Keywords : Deflagration-to-detonation transition (DDT), Obstacle-filled tube, Straight rods, Hydrogen-air mixture, Flame acceleration

\section{Introduction}

Detonation is a combustion mode in which reaction zones coupled to shock waves propagate at a characteristic supersonic speed (Lee, 2008). Moreover, detonation is also characterized by a higher combustion temperature compared with other combustion modes. Taking advantage of the high combustion temperature of detonation, various detonation applications have been proposed (Nikolaev et al., 2003). One such example is a pulse detonation engine (PDE), which is an internal combustion engine (ICE) that generates thrust or power by repeatedly generating detonations in a combustor (Roy et al., 2004). The theoretical thermal efficiency of a PDE (Zel'dovich, 2006) (Endo et al., 2004) is higher than that of conventional ICEs such as gas turbine engines. Accordingly, PDEs have attracted attention as a next-generation ICE. In addition, if detonation is initiated within a short distance, the proportion of combustible gas burned by detonation increases, leading to the improvement of the thermal efficiency of the PDE. However, when a relatively weak energy source such as an automotive spark plug is used as the ignition device for a PDE, detonation is not initiated simultaneously with the ignition of a combustible mixture. In such a case, detonation occurs via the so-called deflagration-to-detonation transition (DDT) (Ciccarelli et al., 2008) (Lee, 2008).

A DDT process is roughly divided into two phases (Ciccarelli et al., 2008) (Lee, 2008): a deflagration wave accelerating to approximately the speed of sound of the burned gas, and a detonation wave actually forming from a microexplosion in the complex of the accelerated flame and shock waves. In most studies that consider shortening the run-up distance to DDT, obstacles were installed in a tube or channel to promote the flame acceleration in the initial phase. A spiral-coil known as the Shchelkin spiral (Shchelkin and Troshin, 1965) or orifice plates have been frequently used as obstacles (Lee et al., 1984) (Peraldi et al., 1988) (Alekseev et al., 2001) (Veser et al., 2002) (Vasil'ev, 2012). Furthermore, the roles of these obstacles in the two DDT phases have been clarified to some degree. When orifice plates are continuously arranged in a channel, the flow induced by the flame repeatedly experiences contraction and expansion. These periodic contractions and expansions cause flame folding, resulting in flame acceleration (Ciccarelli et al., 2005). By contrast, strong shock waves are formed by an accelerating flame, and reflected by the obstacles (Gamezo et al., 2008). Then, the reflected shock waves generate hot regions that produce detonation via the Zel'dovich gradient 
mechanism (Zel'dovich et al., 1970). Moreover, diffracted shock waves are reflected from the side wall of a tube, producing Mach stems. The Mach stems then collide with the obstacles and trigger detonation (Gamezo et al., 2008). In recirculation zones developing downstream of the obstacles, the flame is engulfed and then accelerates and creates a shock wave. The newly formed shock waves are reflected from the next obstacle, and then the shock waves interact with the flame, resulting in the onset of detonation (Obara et al., 2012).

When applying the DDT promotion technique using obstacles in a PDE, the obstacles installed in the PDE will become extremely hot during the PDE operation if the obstacles are not cooled adequately, and then when fresh gas (unburned explosive gas) is supplied to the PDE, the fresh explosive gas may auto-ignite through contact with the hot obstacles. If such an auto-ignition occurs, the pulse-detonation cycle stops and the operation fails. Therefore, the obstacles used for DDT promotion in a PDE should have a structure that can be cooled to prevent auto-ignition. In addition, if recirculation zones of hot burned gas are formed downstream of the obstacles, the fresh explosive gas may be mixed with the hot burned gas in the recirculation zones, and then auto-ignition may occur. Therefore, the obstacles should have a structure that minimizes the downstream recirculation zones. From the above reasons, for the DDT-promoting obstacles for a PDE, an obstacle configuration was adopted in which straight rods were orthogonally alternated in the cylindrical tube. For water-cooling of the obstacles, the straight rods are replaced with straight pipes, through which water flows. Furthermore, by inclining the obstacle pipes at $45^{\circ}$ from the gravitational direction, the cooling water flows inside the obstacle pipes by natural convection.

Although there have been many studies on shortening the DDT run-up distance by using a Shchelkin spiral or orifice plates (Lindstedt and Michels, 1989) (Ciccarelli and Witt, 2006) (Sorin et al., 2006) (Silvestrini et al., 2008), no useful data could be found for optimizing the obstacle rods or pipes orthogonally alternated in a cylindrical tube for DDT promotion because a straight rod perpendicular to the central axis of the tube is topologically different from a Shchelkin spiral or an orifice plate. Therefore, in the present work, we performed detonation-initiation tests with varying spacing, blockage ratio, and the number of obstacle rods to examine improved conditions of the straight rods for DDT promotion. In addition, we varied the equivalence ratio of the explosive mixture in the experiments for examining the effect of the fluctuation of the equivalence ratio during long-time PDE operation.

\section{Experimental apparatus}

Figure 1 shows the schematic diagram of the experimental setup. The apparatus was a cylindrical detonation tube with an inner diameter of $20 \mathrm{~mm}$ consisting of a 700 - $\mathrm{mm}$-long test tube and a 1000-mm-long smooth tube. Both tubes were made of stainless steel (SUS 304). To promote DDT, we installed obstacle rods in the upstream region of the test tube, varying the number of rods, their spacing, and their blockage ratio $(B R)$. Figure 2 illustrates the cross sections of the test tube. The test tube had one hole to install a screwed-type obstacle rod in each cross section as shown in Fig. 2(a). A spacer made of brass was inserted into the hole in test cases in which an obstacle rod was not installed so that the inner surface of the test tube as smooth as possible as shown in Fig. 2(b). However, the tip of the spacer was flat instead of curved along the inside surface of the test tube. The obstacle-rod geometry conditions are summarized in Table 1 . The blockage ratio $(B R)$ is defined as $B R=D d /\left[\pi(D / 2)^{2}\right]$, where $D$ and $d$ are the inner diameter of the test tube and the diameter of the obstacle rod, respectively. The explosive gas used in the present study was a premixed hydrogen-air gas mixture with an equivalence ratio varied from 0.7 to 1.2 at intervals of 0.1 , with an initial pressure of $100 \mathrm{kPa}$ and at room temperature.

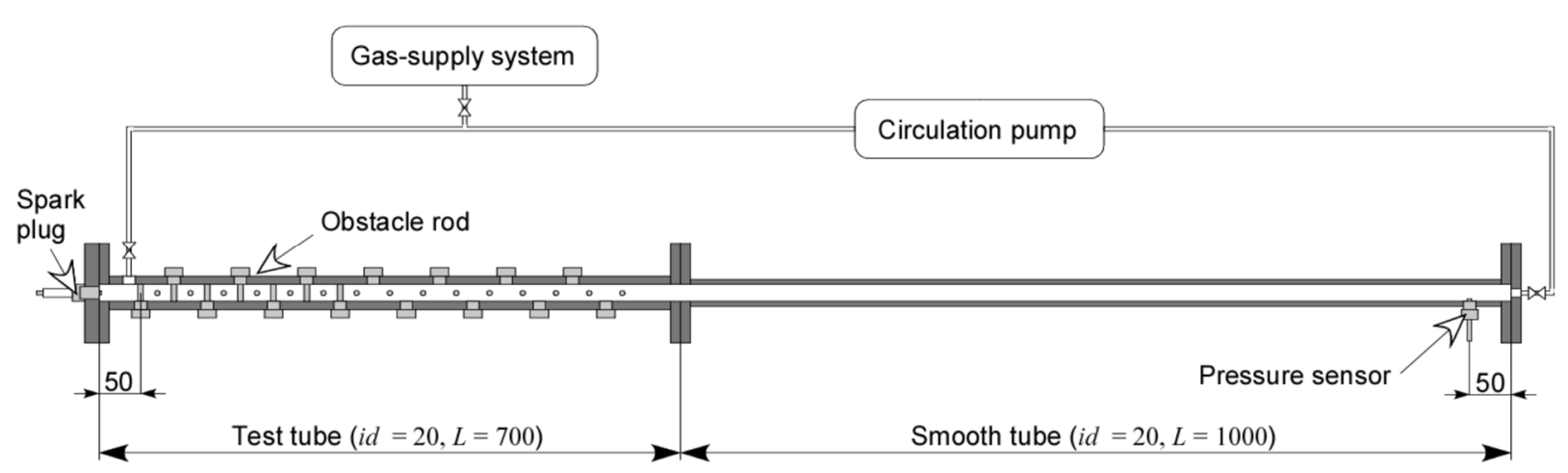

Fig. 1. Experimental apparatus. 
The explosive gas mixture was ignited using a commercially available automobile spark plug (NGK, BKR5E), which was activated by an automobile ignition driver (DENSO, NCP13), installed on the end-wall of the test tube as shown in Fig. 1. To obtain the flame speed, the times when the flame passed through six of the ion probes shown in Fig. 3 were measured, and Table 2 shows the ion probes actually used in each experiment. In addition, a piezoelectric-type pressure transducer and an ion probe were installed at $50 \mathrm{~mm}$ from the end-wall of the smooth tube, namely at a7 or b7 in Fig. 3 , to confirm that the shock wave and the combustion wave reached the end-wall simultaneously.

It has been reported that detonations propagate in an obstacle-filled tube at $80 \%$ of the Chapman-Jouguet (CJ) detonation speed $\left(D_{\mathrm{CJ}}\right.$ ) (Lee et al., 1985), and such a detonation is called a quasi-detonation. It was also reported that when the obstacle region was longer than the required length for detonation initiation, a quasi-detonation propagated at $85 \%$ of $D_{\mathrm{CJ}}$ until it passed through the obstacle region, and subsequently, the detonation recovered from the quasidetonation (Sorin et al., 2006). Based on such results, in the present study, we judged that a detonation occurred when the measured flame speed $V$ was greater than $0.85 D_{\mathrm{CJ}}$. Experiments were repeated three times under every experimental condition, and we determined that a detonation did not occur when the measured flame speed $V$ was less than $0.85 D_{\mathrm{CJ}}$ even once in the repeated experiments to consider detonation applications rather than explosion safety. The CJ detonation speed $D_{\mathrm{CJ}}$ was calculated by using NASA Chemical Equilibrium with Applications (CEA) (Gordon and McBride, 1996) assuming an initial temperature of $300 \mathrm{~K}$.

(a)

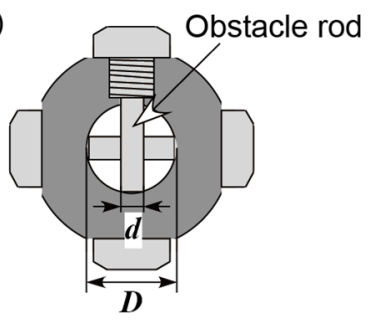

Fig. 2. Cross sections of the test tube. (b)

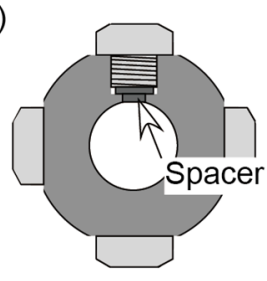

Table 1 Geometric conditions of the obstacle rods.

\begin{tabular}{cc}
\hline Spacing $l[\mathrm{~mm}]$ & \multicolumn{1}{c}{ Diameter $d[\mathrm{~mm}](B R)$} \\
\hline \multirow{2}{*}{20} & $5.0(0.32)$ \\
\cline { 2 - 2 } & $6.5(0.41)$ \\
\hline \multirow{2}{*}{10} & $8.0(0.51)$ \\
\cline { 2 - 2 } & $5.0(0.32)$ \\
\hline
\end{tabular}

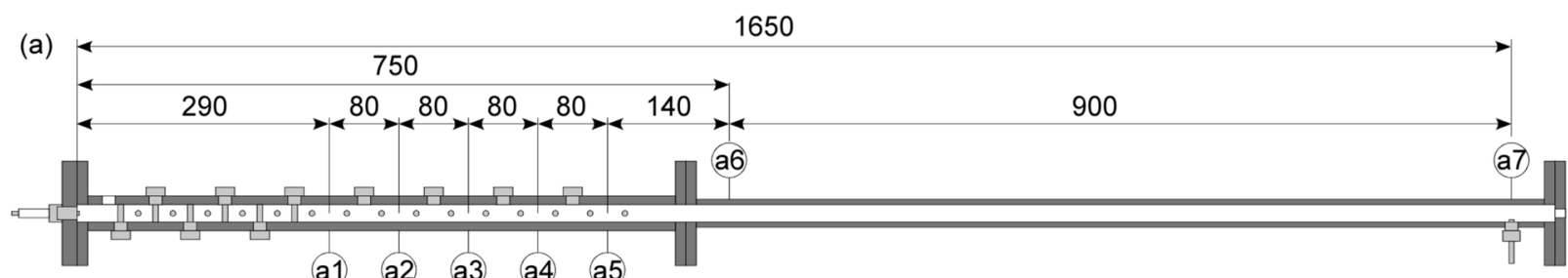

1650

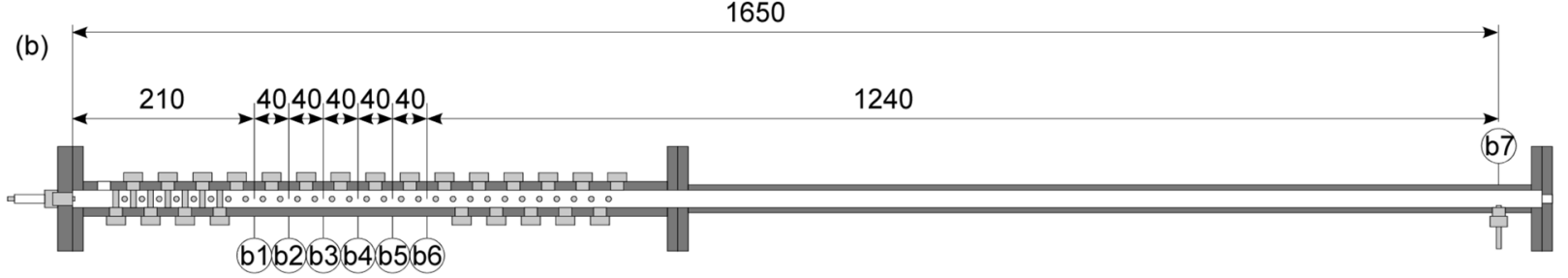

Fig. 3. The positions of the ion probes used in the experiments for the cases of (a) $l=20 \mathrm{~mm}$ and (b) $l=10 \mathrm{~mm}$.

Table 2 The positions of the ion probes from the ignition source.

\begin{tabular}{ccc}
\hline Inter-rod spacing [mm] & Number of rods & Ion-probe positions [mm] \\
\hline \multirow{2}{*}{20} & $9,10,11,12$, & $290(\mathrm{a} 1), 370(\mathrm{a} 2), 450(\mathrm{a} 3), 530(\mathrm{a} 4), 610(\mathrm{a} 5), 1650(\mathrm{a} 7)$ \\
\cline { 2 - 3 } & $13,14,15$ & $370(\mathrm{a} 2), 450(\mathrm{a} 3), 530(\mathrm{a} 4), 610(\mathrm{a} 5), 750(\mathrm{a} 6), 1650(\mathrm{a} 7)$ \\
\hline \multirow{2}{*}{10} & $14,15,16$ & $210(\mathrm{~b} 1), 250(\mathrm{~b} 2), 290(\mathrm{~b} 3), 330(\mathrm{~b} 4), 370(\mathrm{~b} 5), 1650(\mathrm{~b} 7)$ \\
\cline { 2 - 3 } & $17,18,19,20$ & $250(\mathrm{~b} 2), 290(\mathrm{~b} 3), 330(\mathrm{~b} 4), 370(\mathrm{~b} 5), 410(\mathrm{~b} 6), 1650(\mathrm{~b} 7)$ \\
\hline
\end{tabular}




\section{Results and discussion}

\subsection{Determination of suitable obstacle-rod condition for PDE}

An example of the time histories of the ion-probe outputs measured at several positions and the pressure history measured at a7 is shown in Fig. 4. Also, the $x-t$ diagram showing the flame propagation is shown in Fig. 4 . Figure 5 summarizes the results of the experiments for the stoichiometric hydrogen-air mixture for an obstacle rod spacing of $l=20 \mathrm{~mm}$, where "NO GO" and "GO" mean that the minimum flame speed measured in the three-time-repeated experiments was less than and greater than $0.85 D_{\mathrm{CJ}}$, respectively. In addition, "OD” means the case that the maximum flame speed measured in the three-time-repeated experiments was greater than $1.1 D_{\mathrm{CJ}}$, that is, the case of overdriven
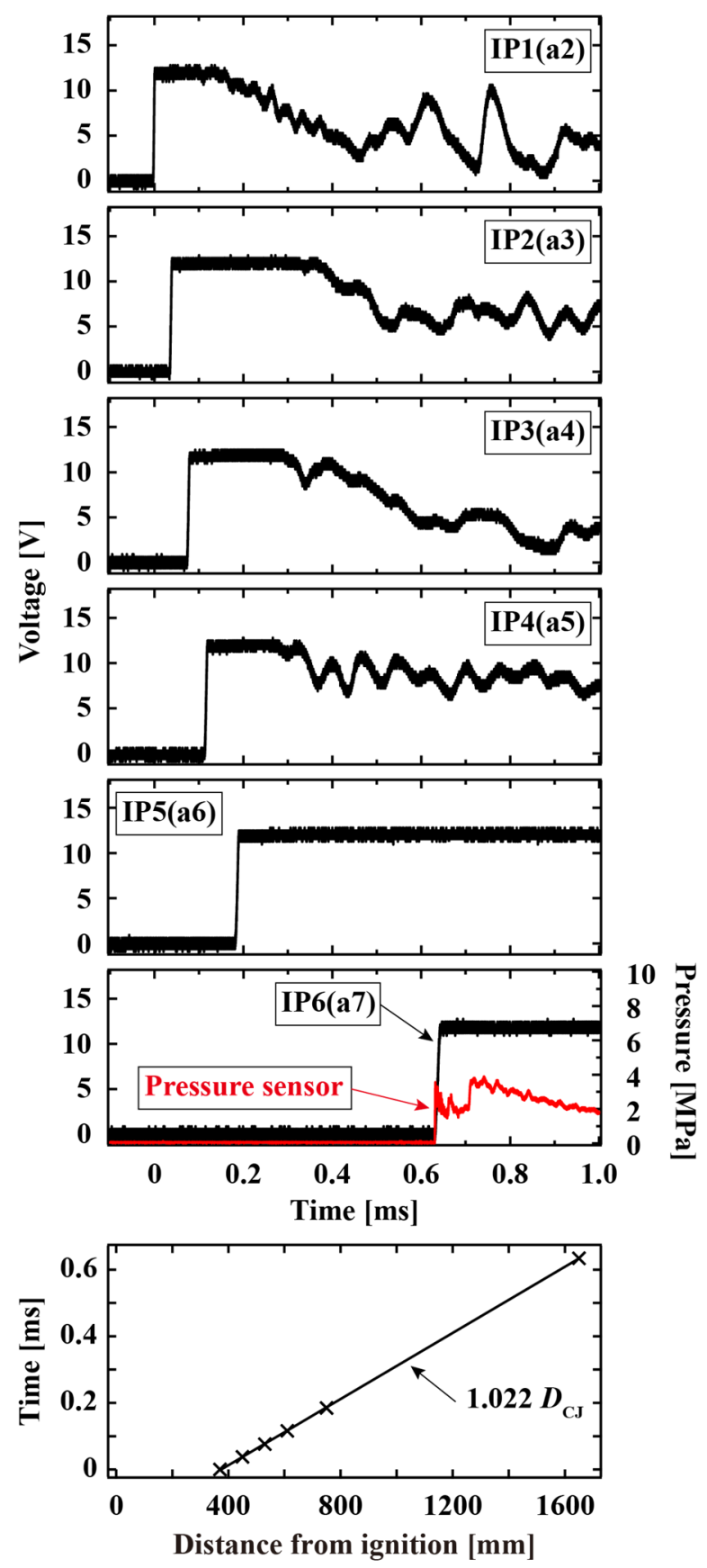

Fig. 4. The time histories of the ion-probe outputs measured at several positions and the pressure history measured at a7 for the case of $l=20 \mathrm{~mm}, B R=0.32, N_{\text {rod }}=14$, and the stoichiometric hydrogen-air mixture. The time origin corresponds to the rise of the output signal of IP1(a2). Also, the $x-t$ diagram showing the flame propagation is shown. 
detonation. Furthermore, the values of $V / D_{\mathrm{CJ}}$ shown in the figures show the average flame speed $V$ of the threetime-repeated experiments between the last two ion probes (a5 or a6-a7, or b5 or b6-b7) normalized by the CJ detonation speed $D_{\mathrm{CJ}}$. The cases in which the value of $V / D_{\mathrm{CJ}}$ was greater than 0.85 in spite of "NO GO," correspond to the case that the detonation onset occurred in the smooth-tube portion downstream of the obstacle region. When the number of rods $N_{\text {rod }}$ was between 13 and 15, detonation propagated already in the first measurement section out of the obstaclerod region regardless of $B R$. Considering that the smaller $B R$ results in the smaller flow resistance as long as $N_{\text {rod }}$ is the same, the case of $B R=0.32$ and $N_{\text {rod }}=13$ is the most suitable for a PDE when $l=20 \mathrm{~mm}$.

Figure 6 summarizes the results of the experiments for the stoichiometric hydrogen-air mixture for an obstacle rod spacing of $l=10 \mathrm{~mm}$. When $B R=0.32$ and $N_{\text {rod }}$ was $16-19$, the detonations propagated already in the first measurement section out of the obstacle-rod region. However, when $B R$ was 0.41 or 0.51 , detonations were not

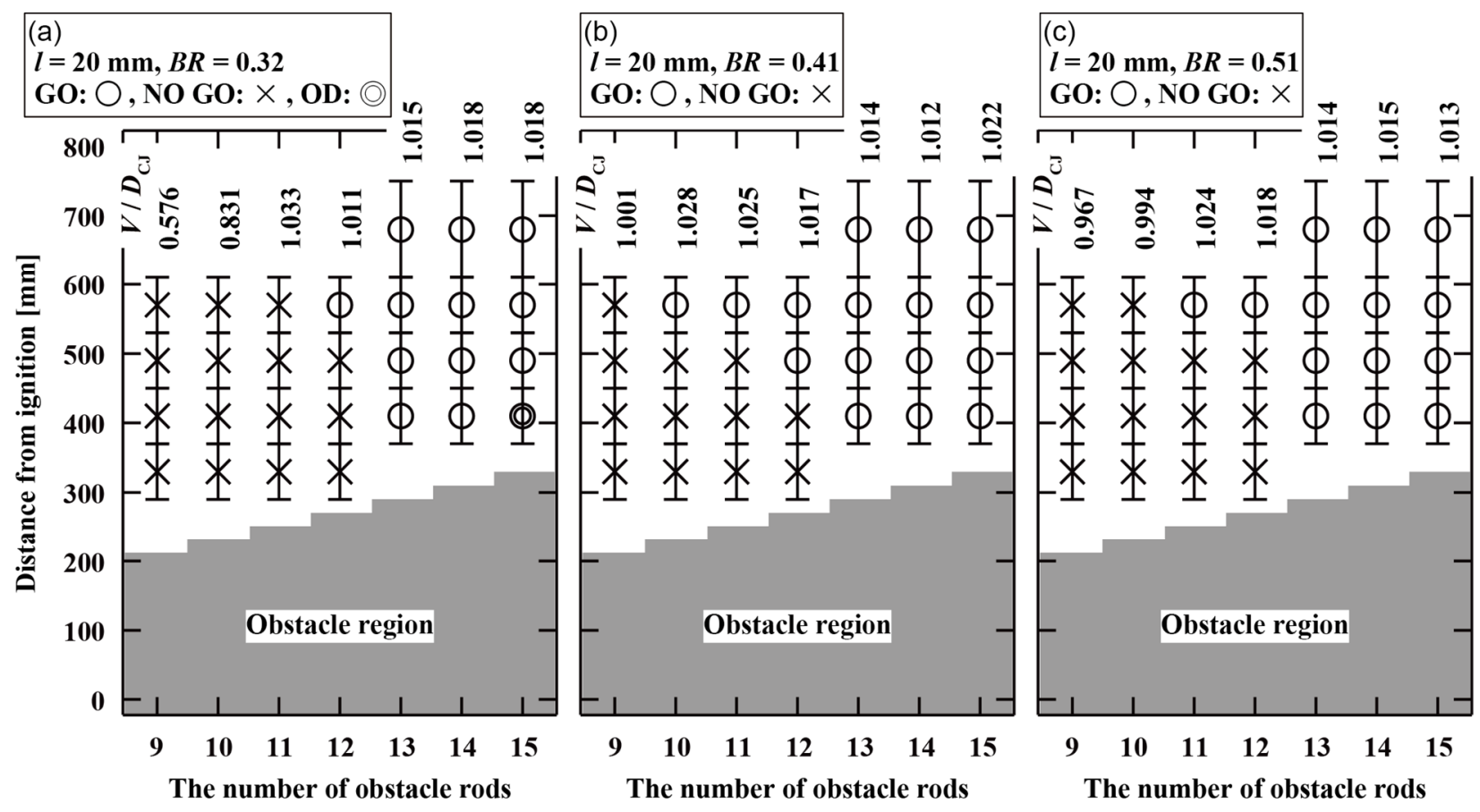

Fig. 5. Experimental results for the stoichiometric hydrogen-air mixture for $l=20 \mathrm{~mm}$.

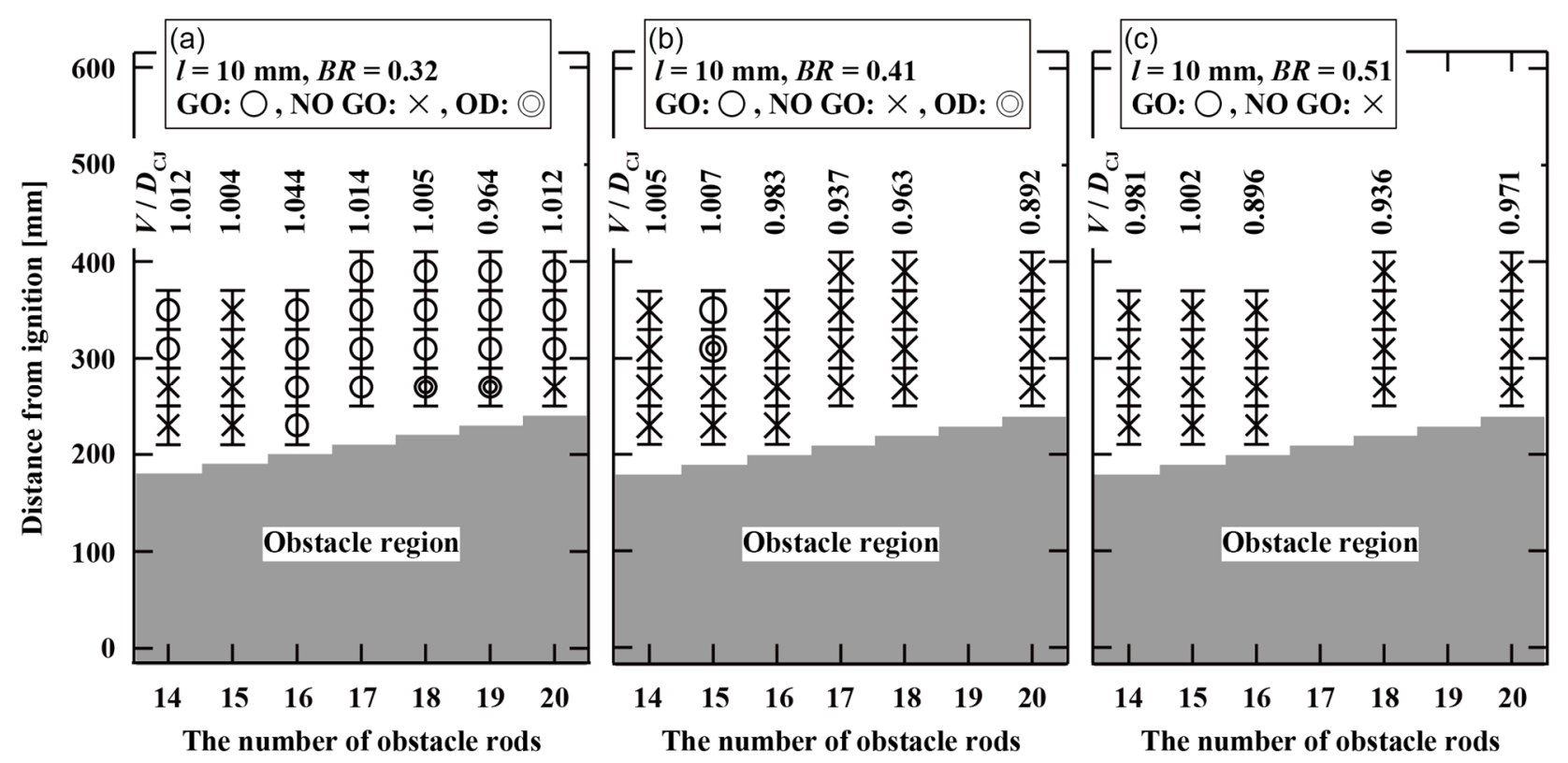

Fig. 6. Experimental results for the stoichiometric hydrogen-air mixture for $l=10 \mathrm{~mm}$. 
observed in the first measurement section out of the obstacle-rod region. It should be noted that in the cases of $B R=0.32, N_{\text {rod }}=14$, and $B R=0.41, N_{\text {rod }}=15$, shown in Figs. 6(a) and 6(b), the detonation onset occurred approximately $100 \mathrm{~mm}$ outside the obstacle region. These cases show ordinary detonation onset. That is, the flame was accelerated in the obstacle region, but the obstacle region was not long enough for DDT. The flame accelerated to some degree in the obstacle region was further accelerated outside the obstacle region by the usual turbulent flame acceleration mechanism. And finally, the detonation onset occurred by the interaction between the flame, the preceding shock wave, and the boundary layer on the side wall of the smooth detonation tube.

In the case of $l=10 \mathrm{~mm}$ and $B R=0.32$ shown in Fig. 6(a), the detonation onset in the obstacle region was observed by increasing the number of rods to $N_{\text {rod }}=16$, although in the cases that $B R$ was 0.41 or 0.51 shown in Fig. $6(\mathrm{~b})$ or 6(c), the detonation onset in the obstacle region was not observed even when the number of rods was increased to $N_{\text {rod }}=20$. On the other hand, in the cases of $l=20 \mathrm{~mm}, B R=0.32,0.41,0.51$, the detonation onset seemed less sensitive to $B R$. That is, the influences of the obstacle $B R$ on DDT were clearly different between the cases of $l=20 \mathrm{~mm}$ and $l=10 \mathrm{~mm}$. And these results suggest that the influence of the obstacle $B R$ on DDT depends on the ratio between the rod diameter $(d)$ to the rod spacing $(l)$, which is another non-dimensional quantity regarding the obstacles. Although details are not clear currently, we think as follows. When the ratio $d / l$ is small, each rod interacts with the gas flow individually. However, when the ratio $d / l$ becomes close to unity, a group of rods interact with the gas flow collectively. In the present experiments, $d / l=0.25,0.325,0.4$ for the cases of $l=20 \mathrm{~mm}$, $B R=0.32,0.41,0.51$, respectively. On the other hand, $d / l=0.5,0.65,0.8$ for the cases of $l=10 \mathrm{~mm}$, $B R=0.32,0.41,0.51$, respectively. The parameter regions of $d / l$ are clearly different between the cases of $l=20 \mathrm{~mm}$ and $l=10 \mathrm{~mm}$. We think that this difference in the ratio $d / l$ is a cause of the different behavior of the influence of the obstacle $B R$ on DDT between the cases of $l=20 \mathrm{~mm}$ and $l=10 \mathrm{~mm}$.

For greater clarification of the obstacle-rod conditions suitable for a PDE, we examined the effects of the equivalence ratio on the DDT promotion by the obstacle rods under some conditions under which good results were obtained for the stoichiometric case. For the case of $l=20 \mathrm{~mm}$, we selected obstacle-rod conditions of $B R=0.32$ and an $N_{\text {rod }}$ of either 13 or 14 . By contrast, for the case of $l=10 \mathrm{~mm}$, we selected $B R=0.32$ and an $N_{\text {rod }}$ ranging 16-19. Figure 7 summarizes the results of the experiments for various equivalence ratios for $l=20 \mathrm{~mm}$ and $B R=0.32$. When $N_{\text {rod }}=14$, detonations were observed already in the first measurement section out of the obstacle-rod region for equivalence ratios ranging 0.8-1.2, although the run-up distance to DDT became remarkably longer for the fuel-lean conditions when $N_{\text {rod }}=13$. Figure 8 summarizes the results of the experiments for various equivalence ratios for $l=10 \mathrm{~mm}$ and $B R=0.32$. Only when $N_{\text {rod }}=19$, detonations were observed already in the first measurement section out of the obstacle-rod region for equivalence ratios ranging $0.8-1.2$, although detonations were not observed in the fuel-
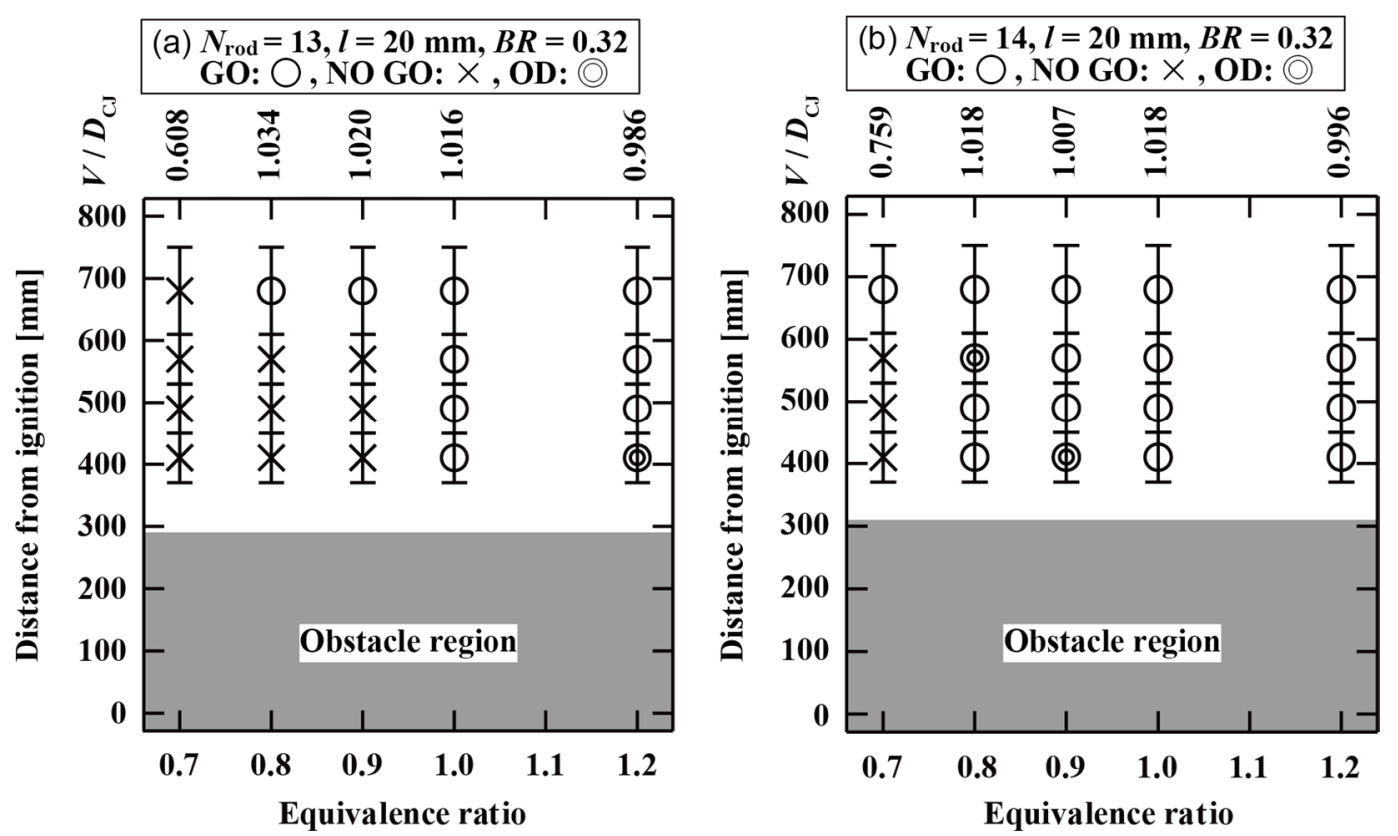

Fig. 7. Experimental results for various equivalence ratios for $l=20 \mathrm{~mm}$ and $B R=0.32$. 
lean conditions for the other values of $N_{\text {rod }}$.

In the case of $l=20 \mathrm{~mm}$, the most suitable obstacle-rod condition for a PDE is that of $B R=0.32$ and $N_{\text {rod }}=14$. In this case, the run-up distance to DDT was approximately $400 \mathrm{~mm}$, corresponding to 20 times the tube diameter, as shown in Fig. 7(b). By contrast, in the case of $l=10 \mathrm{~mm}$, the most suitable obstacle-rod condition for a PDE is that of $B R=0.32$ and $N_{\text {rod }}=19$. In this case, the run-up distance to DDT was approximately $280 \mathrm{~mm}$, corresponding to 14 times the tube diameter, as shown in Fig. 8(d). When this straight-rod-obstacle DDT-promotion technique is applied to a PDE, the smaller number of the obstacle rods may result in a lower probability of their malfunction. In addition, the difference in the run-up distance to DDT of six times the tube diameter between the case of $B R=0.32$ and $N_{\text {rod }}=19$ and the case of $B R=0.32$ and $N_{\text {rod }}=14$ is much smaller than the total combustor length, which is typically 40 times the tube diameter (Takahashi et al., 2012). Based on such considerations, we finally selected the obstacle-rod condition of $l=20 \mathrm{~mm}, B R=0.32$, and $N_{\text {rod }}=14$ as the most suitable one for a PDE.

Let us describe the DDT run-up distance in the published papers briefly. Blanchard et al. (2011) investigated the DDT run-up distance using a smooth cylindrical tube with an inner diameter of $159 \mathrm{~mm}$. The gas condition was the stoichiometric hydrogen-air mixture with an initial pressure of 1 bar. As a result, the DDT run-up distance was $11.84 \mathrm{~m}$ corresponding to approximately 75 times the tube diameter. Because the ignition energy in their experiments was $1-10$ $\mathrm{J}$, the DDT run-up distance will be longer if an automotive spark plug, whose ignition energy is typically a few tens millijoules, is used like the present experiments. In addition, the ability of shortening the DDT run-up distance of the obstacle rods is compared with those of the Shchelkin spiral or orifice plates. Sorin et al. (2006) investigated the DDT run-up distance by an experimental device in which the Shchelkin spiral with $B R=0.52$ and a pitch of $l=26 \mathrm{~mm}$
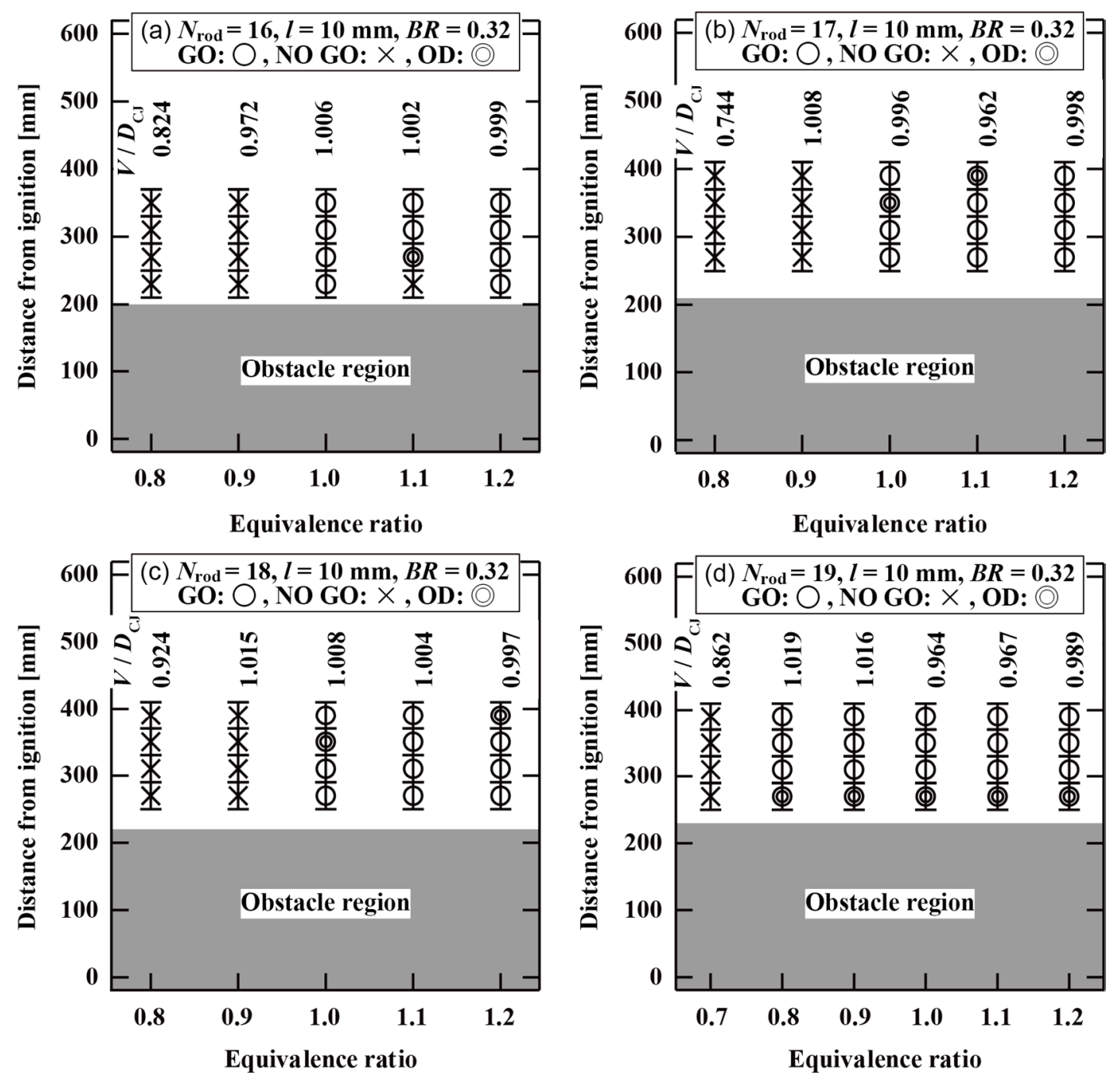

Fig. 8. Experimental results for various equivalence ratios for $l=10 \mathrm{~mm}$ and $B R=0.32$. 
was inserted into a cylindrical tube with an inner diameter of $D=26 \mathrm{~mm}$. The gas condition was $\mathrm{H}_{2}+0.5 \mathrm{O}_{2}+1.88 \mathrm{~N}_{2}$ with an initial pressure of $p_{0}=1 \mathrm{bar}$ and an initial temperature of $T_{0}=273 \mathrm{~K}$. As a result, the DDT run-up distance $L_{\mathrm{DDT}}$ was $366 \mathrm{~mm}$ corresponding to 14 times the tube diameter. Card et al. (2005) investigated the DDT run-up distance by an experimental device in which orifice plates with $B R=0.43$ and a pitch of $l=100 \mathrm{~mm}$ were inserted into a cylindrical tube with an inner diameter of $D=100 \mathrm{~mm}$. The gas condition was the stoichiometric hydrogen-air mixture with an initial pressure of $p_{0}=1 \mathrm{~atm}$ and an initial temperature of $T_{0}=300 \mathrm{~K}$. As a result, the DDT run-up distance $L_{\mathrm{DDT}}$ was approximately $750 \mathrm{~mm}$ corresponding to 7.5 times the tube diameter. The results of the present experiments are that $L_{\mathrm{DDT}}$ was approximately 20 times the tube diameter for the case of $l=20 \mathrm{~mm}$ and approximately 14 times the tube diameter for the case of $l=10 \mathrm{~mm}$ and $B R=0.32$. From the above, the ability of shortening the DDT run-up distance of the obstacle rods in the present study is somewhat inferior to that of orifice plates but is almost comparable to that of the Shchelkin spiral.

\subsection{Criterion for the detonation initiation in the obstacle-rod-filled tube}

Figures 7(b) and 8(d) suggest that there is a criterion for the equivalence ratio for the detonation onset. Dorofeev et al. (2000) proposed a characteristic length $L$ required for the formation of detonation in a channel with repeated obstacles. This characteristic length $L$ is defined, for a large number of $N_{\text {rod }}$, by

$$
L=\frac{D+l}{2(1-\sqrt{1-B R})},
$$

where $D$ is the diameter of the tube and $l$ is the spacing between the obstacles. In our experiments, these parameters are $D=20 \mathrm{~mm}$ and $l=10$ or $20 \mathrm{~mm}$. Based on the results of many experiments in which a cylindrical tube with orifice plates was primarily used, Dorofeev et al. showed that the criterion for the onset of detonation can be given as $L \geq 7 \lambda$ under the condition that the flame is accelerated sufficiently, where $\lambda$ is the detonation cell width of the test gas. The sufficient flame speed before the detonation onset is approximately half of the CJ detonation speed $D_{\mathrm{CJ}}$, nearly corresponding to the speed of sound of the burned gas.

The value of $L$ calculated for the present experiments is $85.5 \mathrm{~mm}$ for $l=10 \mathrm{~mm}$ and $B R=0.32$, or $114 \mathrm{~mm}$ for $l=20 \mathrm{~mm}$ and $B R=0.32$. The flame speed was measured outside the obstacle region in the present experiments. Strictly speaking, the $L / \lambda \simeq 7$ criterion is applicable to the case that the detonation onset occurs inside the obstacle region. Comparing the distance between the last obstacle rod and the first ion probe with the characteristic length $L$ calculated by eq. (1), the former was shorter than the latter in all cases but the case of $l=20 \mathrm{~mm}, B R=0.51$ shown in Fig. 5(c). Therefore, we judged that the cases in which the flame speed in the first measurement section was expressed by "GO" or "OD" meant that the detonation onset occurred in the obstacle region or its equivalent. The only exception of $l=20 \mathrm{~mm}, B R=0.51$ shown in Fig. 5(c) will be discussed later. Figure 9 shows the ratio between the characteristic length $L$ and the detonation cell width $\lambda$ (Guirao et al., 1982) (Tieszen et al., 1986) (Ciccarelli et al., 1994) for various

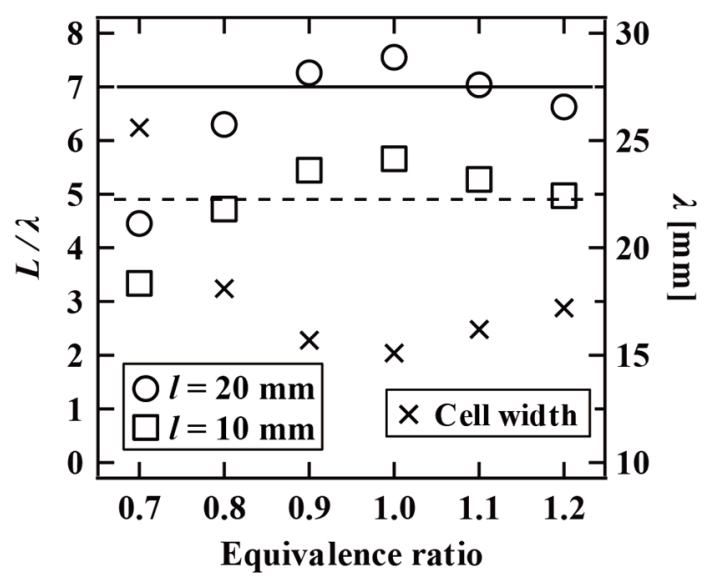

Fig. 9. Evaluated values of $L / \lambda$ and the detonation cell width $\lambda$ for various equivalence ratios. 
equivalence ratios ranging $0.7-1.2$. The solid line corresponds to $L / \lambda=7$, the proposed criterion, and the broken line shows the lower accuracy limit $(-30 \%)$ of the criterion proposed by Dorofeev et al. (2000). As shown in Fig. 9, the values of $L / \lambda$ evaluated for $l=10$ or $20 \mathrm{~mm}$ and $B R=0.32$ satisfy the condition of $L \geq 7 \lambda$ as proposed by Dorofeev et al. when considering an accuracy of $\pm 30 \%$ for the equivalence ratios ranging $0.8-1.2$. However, for the equivalence ratio of $0.7, L / \lambda$ for $l=10$ or $20 \mathrm{~mm}$ and $B R=0.32$ does not satisfy the condition of $L \geq 7 \lambda$. Interestingly, this criterion successfully explains the experimental results shown in Figs. 7(b) and 8(d), suggesting that the criterion proposed by Dorofeev et al. (2000) is applicable to the configuration of the present study. The reason for this applicability is likely that the onset of detonation requires only sufficient space in which a detonation can be triggered by the Zel'dovich mechanism (Zel'dovich et al., 1970) as long as the flame is sufficiently accelerated beforehand.

Finally, the influence of $B R$ on the characteristic length $L$ regarding the detonation onset observed in the present experiments showed that the detonation onset was observed in the case of $L / \lambda=4.4$, where $l=20 \mathrm{~mm}, B R=0.51$, and stoichiometric condition, although the lower limit $(-30 \%)$ of the $L / \lambda \simeq 7$ criterion is 4.9 . Considering the original expression of $L / \lambda \simeq 7$, this is an empirical formula, and the distance between the last obstacle rod and the first ion probe was longer than the characteristic length $L$ calculated by eq. (1) only in this case, the result of this case is not considered to negate the conclusion that the $L / \lambda \simeq 7$ criterion on the detonation onset is applicable to the present experiments.

\section{Conclusion}

We experimentally investigated the promotion of deflagration-to-detonation transition (DDT) in hydrogen-air mixtures contained in a tube equipped with straight-shaped rods as obstacles. In the experiments, the number of obstacle rods, their spacing, their blockage ratio, and the equivalence ratio of the hydrogen-air mixture were varied as the governing parameters. As a result of an optimization to some degree of the obstacle-rod conditions, the run-up distance to DDT was shortened down to 20 times the tube diameter for a blockage ratio and spacing of the obstacle rods of 0.32 and $20 \mathrm{~mm}$, respectively, for hydrogen-air mixtures with equivalence ratios ranging $0.8-1.2$. In addition, it was found that the criterion for the detonation onset proposed by Dorofeev et al. (2000) is applicable to the experimental conditions of the present study.

\section{Acknowledgements}

This work was supported by JSPS KAKENHI Grant Number JP17H03482. The authors are grateful to Kohei Imoto for his assistance in the experiments.

\section{References}

Alekseev, V. I., Kuznetsov, M. S., Yankin, Yu. G. and Dorofeev, S. B., Experimental study of flame acceleration and the deflagration-to-detonation transition under conditions of transverse venting, Journal of Loss Prevention in the Process Industries, Vol. 14 (2001), pp. 591-596.

Blanchard, R., Arndt, D., Gratz, R. and Scheider, S., Effect of ignition position on the run-up distance to DDT for hydrogen-air explosions, Journal of Loss Prevention in the Process Industries, Vol. 24 (2011), pp. 194-199.

Card, J., Rival, D. and Ciccarelli, G., DDT in fuel-air mixtures at elevated temperatures and pressures, Shock Waves, Vol. 14 (2005), pp. 167-173.

Ciccarelli, G., Ginsberg, T., Boccio, J., Economos, C., Sato, K. and Kinoshita, M., Detonation cell size measurements and predictions in hydrogen-air-steam mixtures at elevated temperatures, Combustion and Flame, Vol. 99 (1994), pp. 212-220.

Ciccarelli, G., Flower, C. J. and Bardon, M., Effect of obstacle size and spacing on the initial stage of flame acceleration in a rough tube, Shock Waves, Vol. 14 (2005), pp. 161-166.

Ciccarelli, G. and Witt, B. de, Detonation initiation by shock reflection from an orifice plate, Shock Waves, Vol. 15 (2006), pp. 259-265.

Ciccarelli, G. and Dorofeev, S. B., Flame acceleration and transition to detonation in ducts, Progress in Energy and Combustion Science, Vol. 34 (2008), pp. 499-550.

Dorofeev, S. B., Sidorov, V. P., Kuznetsov, M. S., Matsukov, I. D. and Alekseev, V. I., Effect of scale on the onset of 
detonations, Shock Waves, Vol. 10 (2000), pp. 137-149.

Endo, T., Yatsufusa, T., Taki, S., and Kasahara, J., Thermodynamic analysis of the performance of a pulse detonation turbine engine, Science and Technology of Energetic Materials, Vol. 65 (2004), pp. 103-110 (in Japanese).

Gamezo, V. N., Ogawa, T. and Oran, E. S., Flame acceleration and DDT in channels with obstacles: Effect of obstacle spacing, Combustion and Flame, Vol. 155 (2008), pp. 302-315.

Gordon, S. and McBride, B. J., NASA Reference Publication 1311 (1996).

Guirao, C. M., Knystautas, R., Lee, J. H. S., Benedick, W. and Berman, M., Hydrogen-air detonations, Proceedings of the Combustion Institute, Vol. 19 (1982), pp. 583-590.

Lee, J. H. S., Knystautas, R. and Freiman, A., High speed turbulent deflagrations and transition to detonation in $\mathrm{H}_{2}$-air mixtures, Combustion and Flame, Vol. 56 (1984), pp. 227-239.

Lee, J. H. S., Knystautas, R. and Chan, C. K., Turbulent flame propagation in obstacle-filled tubes, Proceedings of the Combustion Institute, Vol. 20 (1985), pp. 1663-1672.

Lee, J. H. S., The Detonation Phenomenon (2008), Chapters 2 and 8, Cambridge University Press.

Lindstedt R. P. and Michels, H. J., Deflagration to detonation transitions and strong deflagrations in alkane and alkene air mixtures, Combustion and Flame, Vol. 76 (1989), pp. 169-181.

Nikolaev, Yu. A., Vasil'ev, A. A. and Ul'yanitskii, B. Yu., Gas detonation and its application in engineering and technologies, Combustion, Explosion, and Shock Waves, Vol. 39, No. 4 (2003), pp. 382-410.

Obara, T., Kobayashi, T. and Ohyagi, S., Mechanism of deflagration-to-detonation transitions above repeated obstacles, Shock Waves, Vol. 22 (2012), pp. 627-639.

Peraldi, O., Knystautas, R. and Lee, J. H. S., Criteria for transition to detonation in tubes, Proceedings of the Combustion Institute, Vol. 21 (1988), pp. 1629-1637.

Roy, G. D., Frolov, S. M., Borisov, A. A. and Netzer, D. W., Pulse detonation propulsion: challenges, current status, and future perspective, Progress in Energy and Combustion Science, Vol. 30 (2004), pp. 545-672.

Shchelkin, K. I. and Troshin, Ya. K., Gasdynamics of combustion (1965), Mono Book Corporation.

Silvestrini, M., Genova, B., Parisi, G. and Leon Trujillo, F. J., Flame acceleration and DDT run-up distance for smooth and obstacles filled tubes, Journal of Loss Prevention in the Process Industries, Vol. 21 (2008), pp. 555-562.

Sorin, R., Zitoun, R. and Desbordes, D., Optimization of the deflagration to detonation transition: reduction of length and time of transition, Shock Waves, Vol. 15 (2006), pp. 137-145.

Takahashi, T., Mitsunobu, A., Ogawa, Y., Kato, S., Yokoyama, H., Susa, A. and Endo, T., Experiments on energy balance and thermal efficiency of pulse detonation turbine engine, Science and Technology of Energetic Materials, Vol. 73, No. 6 (2012), pp. 181-187.

Tieszen, S. R., Sherman, M. P., Benedick, W. B., Shepherd, J. E., Knystautas, R. and Lee, J. H. S., Detonation cell size measurements in hydrogen-air-steam mixtures, Progress in Astronautics and Aeronautics, Vol. 106 (1986), pp. 205219.

Vasil'ev, A. A., Optimization of the deflagration-to-detonation transition, Combustion, Explosion, and Shock Waves, Vol. 48, No. 3 (2012), pp. 269-277.

Veser, A., Breitung, W. and Dorofeev, S. B., Run-up distances to supersonic flames in obstacle-laden tubes, Journal de Physique IV France, Vol. 12, No. 7 (2002), pp. 333-340.

Zel'dovich, Ya. B., Librovich, V. B., Makhviladze, G. M. and Sivashinsky, G. I., On the development of detonation in a non-uniformly preheated gas, Astronautica Acta, Vol. 15 (1970), pp. 313-321.

Zel'dovich, Ya. B., To the question of energy use of detonation combustion, Journal of Propulsion and Power, Vol. 22 (2006), pp.588-592. 